\title{
Avaliação da dor no recém-nascido
} prematuro: parâmetros fisiológicos versus comportamentais

\author{
Assessment of pain in premature newborns: physiological and behavioral variables
}

Carla Marques Nicolau', Kiliana Modesto', Priscila Nunes' ${ }^{1}$ Karina Araújo ${ }^{1}$, Heloisa Amaral', Mário Cícero Falcão²

\section{Resumo}

Introdução: A dor no período neonatal é motivo de inúmeros estudos, sendo fundamental para a sua abordagem uma avaliação criteriosa e adequada.

Objetivo: Comparar as variáveis fisiológicas com as comportamentais para a avaliação da dor em recém-nascido (RN) prematuro. Métodos: Estudo realizado entre fevereiro de 2003 e maio de 2004, com recém-nascidos de idade gestacional (IG) abaixo de 34 semanas e peso de nascimento menor que $1500 \mathrm{~g}$, submetidos à ventilação mecânica e não sedados. As variáveis fisiológicas estudadas foram as freqüências cardíaca (FC) e respiratória (FR) e a saturação de oxigênio $\left(\mathrm{SatO}_{2}\right)$; as variáveis comportamentais foram avaliadas por meio da escala de dor do recém-nascido - Neonatal Infant Pain Scale (NIPS). Todas as variáveis foram controladas no terceiro dia de vida do RN, antes, imediatamente após e cinco minutos após o procedimento de aspiração endotraqueal. Resultados: Foram estudados 50 RNs com IG média ao nascimento de 29,98 \pm 2,24 semanas e peso médio de nascimento de 1087,20 $\pm 350,06 \mathrm{~g}$, sendo 28 (56\%) RN do sexo feminino e a doença das membranas hialinas foi diagnosticada em todos os RNs estudados. Pelo teste $t$-Student verificou-se diferença significante na $\mathrm{Sat}_{2}$ nos momentos estudados, sendo o mesmo não observado em relação a $\mathrm{FC}$ e $\mathrm{FR}$. A mediana da NIPS foi maior nos momentos pós aspiração. As variáveis fisiológicas mostraram-se pouco sensíveis à detecção da dor (FC: 40,7\%, FR: 24,1\%, $\mathrm{SatO}_{2}: 6,6 \%$ ), e a escala NIPS mostrou-se mais específica para a mesma avaliação (86,6\%). Conclusão: As variáveis fisiológicas apresentaram pouca sensibilidade e especificidade para a avaliação da dor no RN prematuro, quando avaliadas isoladamente.

Palavras-chave: Recém-nascido; dor; avaliação da dor.

\begin{abstract}
Introduction: Pain in the neonatal period is the objective of several studies, being important for its approach an adequate evaluation. Objective: To compare the physiological and behavioral variables with the behavior for assessment of pain in premature newborns (NB). Methods: Prospective study carried out from February/2003 to May/2004, including premature infants with gestational age (GA) below 34 weeks and birth weight less than 1.500 g, submitted to mechanical ventilation and not sedation. The physiological variables studied were: heart rate (HR), respiratory rate (RR) and oxygen saturation $\left(\mathrm{SatO}_{2}\right)$; the behavioral variables were evaluated by NIPS. All the variables have been controlled in the third day of life of NB, immediately after and five minutes after the procedure of endotracheal suction. Results: Fifty premature infants were studied with GA at birth of $29.98 \pm 2.24$ weeks and birth weight $1087.20 \pm 350.06 \mathrm{~g}$ and the respiratory distress syndrome was diagnosed in all NB studied. Using t-Student test, it was verified significant difference on $\mathrm{SatO}_{2}$ at the different moments studied; however, there was no statistical significant difference in relation to HR and RR. The median of NIPS was high on moments following suction. The physiological variables showed: low sensitivity on detection of pain (HR: $40.7 \%, \mathrm{RR}: 24.1 \%$, Sat ${ }_{2}$ : 6.6:\%), and the NIPS scale showed to be more specific in the same assessment (86.6\%). Conclusion: The physiological variables showed low sensitivity and specific for assessment of pain in premature newborns when used alone.
\end{abstract}

Keywords: Infant, newborn; pain; pain measurement.

Recebido: 08/10/2007

Revisado: $18 / 07 / 2008$

Aprovado: 12/08/2008

Serviço de Fisioterapia, Instituto da Criança, Hospital das Clínicas da Faculdade de Medicina da Universidade de São Paulo (USP), São Paulo (SP)

2 Disciplina de Neonatologia, Departamento de Pediatria da USP, São Paulo (SP)

Endereço para correspondência: Carla Marques Nicolau/Avenida Doutor Enéas de Carvalho Aguiar, 647 - Cerqueira César, CEP: $05403-900$ - São Paulo

(SP), E-mail: carla.nicolau@icr.usp.br 


\section{Introdução}

A dor neonatal merece atenção especial, pois esses pacientes não a expressam verbalmente e suas manifestações são distintas das outras faixas etárias. Trabalhos realizados nos últimos anos comprovaram que os recém-nascidos $(\mathrm{RN})$ a termo e os prematuros com mais de 24 semanas de gestação possuem elementos necessários do sistema nervoso central para a transmissão do estímulo doloroso e memória para a dor, respondendo por meio de alterações fisiológicas e comportamentais ${ }^{1-5}$.

Vários indicadores fisiológicos podem ser usados na avaliação, quantificação e qualificação do estímulo doloroso. Essas variáveis incluem: freqüências cardíaca e respiratória, pressão arterial, saturação de oxigênio, sudorese palmar, tônus vagal, entre outras. Porém, tais indicadores fisiológicos não estão especificamente relacionados à dor. As principais reações comportamentais do RN frente à dor são: o choro, a atividade motora e a mímica facial. Apesar das medidas comportamentais serem mais representativas do que os parâmetros fisiológicos para a avaliação da dor do RN, a avaliação comportamental é dependente da interpretação do observador acerca dos comportamentos avaliados ${ }^{2,4-7}$.

Sabe-se que o tratamento adequado da dor neonatal está associado a menores complicações e redução da mortalidade. Nesse contexto, faz-se importante reconhecer os procedimentos geradores de dor, para que estes sejam realizados somente na vigência de sua real necessidade e acompanhados de métodos analgésicos apropriados ${ }^{8-11}$.

Devido ao caráter subjetivo da dor, métodos multidimensionais de avaliação da dor devem ser utilizados. Dentre as várias escalas de dor descritas e validadas a NIPS (Neonatal Infant Pain Scale) tem se mostrado útil para a avaliação da dor em $\mathrm{RN}$ a termo e prematuros, conseguindo diferenciar os estímulos dolorosos dos não dolorosos. A NIPS é composta por seis indicadores de dor, cinco comportamentais e um fisiológico: expressão facial ( 0 ou 1 ponto); choro ( 0,1 ou 2 pontos); movimentação de braços e pernas ( 0 ou 1 ponto); estado de sono/alerta ( 0 ou 1 ponto) e o padrão respiratório ( 0 ou 1 ponto). Considera-se a dor presente quando a pontuação é superior a três ${ }^{1,3,6,12-13}$.

Com a implementação dos processos de humanização nas terapias intensivas é grande a preocupação com a dor neonatal e, conseqüentemente, com instrumentos que possam quantificá-la e métodos seguros de tratá-la e/ou minimizá-la ${ }^{14-17}$.

O presente estudo objetivou comparar as variáveis fisiológicas - freqüências cardíaca e respiratória e saturação de oxigênio - com as variáveis comportamentais por meio da escala NIPS para a avaliação da dor em RN prematuro quando submetido a um estímulo doloroso.

\section{Métodos}

Trata-se de estudo longitudinal prospectivo de uma série de casos, realizado entre fevereiro de 2003 e maio de 2004, na terapia intensiva neonatal do Berçário Anexo à Maternidade do Hospital das Clínicas da
Faculdade de Medicina da USP (HC-FMUSP). A pesquisa foi desenvolvida após análise e aprovação pela Comissão de Ética para Análise de Projetos de Pesquisa da Diretoria Clínica do HC-FMUSP, sob o número do protocolo 1078/02.

Foram incluídos $\mathrm{RN}$ prematuros com idade gestacional menor que 34 semanas e peso de nascimento menor que $1500 \mathrm{~g}$, que necessitavam de ventilação mecânica. Os RN não estavam recebendo analgésicos e/ ou sedativos. Foram excluídos os RN portadores de malformações congênitas e síndromes genéticas.

Os dados foram coletados no terceiro dia de vida de cada RN, após os mesmos terem atingido a estabilidade clínica. O procedimento escolhido e considerado como potencialmente doloroso foi a aspiração endotraqueal e de vias aéreas superiores. Tal procedimento foi realizado somente quando havia quadro de secreção pulmonar que justificasse a sua aplicação. Todos os RN estudados foram submetidos a este procedimento e os dados foram coletados na primeira intervenção da aspiração endotraqueal.

Dois pesquisadores estavam envolvidos no estudo: um executava o procedimento de aspiração e o outro pesquisador observava os parâmetros comportamentais e as variáveis fisiológicas relacionadas à presença de dor. Um sensor de oxímetro foi fixado na planta do pé do RN para a avaliação das variáveis fisiológicas.

As variáveis fisiológicas estudadas foram: a freqüência cardíaca, a freqüência respiratória e a saturação de oxigênio. A freqüência respiratória foi mensurada por meio de um cronômetro; a saturação de oxigênio e a freqüência cardíaca foram avaliadas por meio de um oxímetro de pulso, sendo considerado somente quando o sinal emitido pelo oxímetro era de bom padrão. Considerou-se como bradicardia, freqüência cardíaca (FC) inferior a 80 batimentos por minuto (bpm) e taquicardia, FC superior a 160 bpm. Em relação à freqüência respiratória (FR), foi considerada bradipnéia, FR inferior a 40 respirações por minuto (rpm), e taquipnéia, FR superior a $60 \mathrm{rpm}$. Definiu-se a presença de hipóxia quando a saturação de oxigênio obtida foi inferior a $90 \%$. Os parâmetros comportamentais foram avaliados segundo a escala NIPS. Tanto os parâmetros fisiológicos quanto a escala NIPS foram aplicados em dois momentos distintos: antes e após a aspiração endotraqueal e de vias aéreas superiores.

Para a análise estatística foram utilizados os testes $t$-Student $\mathrm{e}$ ANOVA one-way, considerando-se p $<0,05$ como nível de significância. Também foram calculados os valores da sensibilidade, da especificidade e os valores preditivos positivo e negativo das variáveis fisiológicas e comportamentais. Os dados foram processados no software Sigma Stat (Sigma Stat ${ }^{\circledR}$ Statistical Software User's Manual, 2002).

\section{Resultados}

A população estudada constituiu-se de 50 recém-nascidos, sendo 22 (44\%) do sexo masculino e 28 (56\%) do sexo feminino. Os dados sobre as características gerais dos RN estão apresentados na Tabela 1. 
Tabela 1 - Características da população estudada

\begin{tabular}{lc}
\hline Característica & População $(\mathrm{n}=50)$ \\
\hline Peso (gramas) & $1087,20 \pm 350,06$ \\
\hline Idade gestacional (semanas) & $29,98 \pm 2,24$ \\
Apgar 5 $5^{\circ}$ minuto $<5 \mathrm{n}(\%)$ & $5(10 \%)$ \\
Sexo $\mathrm{n}(\%)$ & \\
$\quad$ Masculino & $22(44 \%)$ \\
$\quad$ Feminino & $28(56 \%)$ \\
Adequação nutricional $\mathrm{n}(\%)$ & $32(64 \%)$ \\
$\quad$ Adequado idade gestacional & $18(36 \%)$ \\
\hline \multicolumn{1}{c}{ Pequeno idade gestacional } & $72 \pm 12$ \\
\hline Idade pós-natal (horas) &
\end{tabular}

Tabela 2 - Valores da freqüência cardíaca, freqüência respiratória e da saturação de oxigênio e da escala de NIPS de acordo com o momento estudado

\begin{tabular}{lcccc}
\hline Momento & FC (bpm) & FR (rpm) & Sat02 (\%) & NIPS \\
\cline { 2 - 5 } Estudado & Média DP & Média DP & Média DP & Mediana \\
\hline T0 & $150,52 \pm 19,45$ & $51,76 \pm 13,11$ & $95,64 \pm 2,46$ & $2(0-5)$ \\
T1 & $155,04 \pm 17,96$ & $49,84 \pm 12,80$ & $91,42 \pm 3,18$ & $4(0-6)$ \\
T5 & $151,10 \pm 16,09$ & $50,00 \pm 10,43$ & $96,12 \pm 2,66$ & $3(0-6)$ \\
\hline
\end{tabular}

FC: freqüência cardíaca; FR: freqüência respiratória; Sat02: saturação de oxigênio; DP: Desvio padrão; T0: antes da aspiração endotraqueal; T1: imediatamente após a aspiração endotraqueal; T5: cinco minutos após a aspiração endotraqueal.

FC (TOxT1xT5): ANOVA one-way $\mathrm{p}=0,44$

FR (TOxT1xT5): ANOVA one-way $\mathrm{p}=0,86$

$\mathrm{SatO}_{2}$ (TOxT1xT5): K-Wallis one-way $\mathrm{p}<0,001$

Sat $0_{2}(\mathrm{TOxT1})$ e (T1xT5) $\mathrm{p}<0,05$

NIPS (TOxT1xT5): K-Wallis one-way $\mathrm{p}<0,001$

NIPS (TOxT1) e (T1xT5) $p<0,05$

Tabela 3 - Número de RN com bradicardia e/ou taquicardia, bradipnéia e/ou taquipnéia, com hipóxia e com dor (pelo escore de NIPS) nos diferentes momentos estudados

\begin{tabular}{llll}
\hline & T0 & T1 & T5 \\
\hline FC $<80$ e/ou $>160 \mathrm{bpm}$ & $13(26 \%)$ & $23(46 \%)$ & $14(28 \%)$ \\
FR $<40 \mathrm{e} / \mathrm{ou}>60 \mathrm{rpm}$ & $13(26 \%)$ & $15(30 \%)$ & $11(22 \%)$ \\
$\mathrm{SatO}_{2}<90 \%$ & $1(2 \%)$ & $10(20 \%)$ & $2(4 \%)$ \\
$\mathrm{NIIS}>3$ & $4(8 \%)$ & $26(52 \%)$ & $21(40 \%)$ \\
\hline
\end{tabular}

RN: recém-nascido; FC: freqüência cardíaca; bpm: batimentos por minuto; FR; freqüência respiratória; rpm: respirações por minuto; Sat02: saturação de oxigênio; NIPS: escala de dor para o recém-nascido.

FC (TOxT1xT5): ANOVA one-way $\mathrm{p}=0,33$

FR (TOxT1xT5): ANOVA one-way $p=0,16$

$\mathrm{Sat}_{2}$ (T0xT1xT5): K-Wallis one-way $\mathrm{p}<0,001$

SatO $_{2}$ (TOxT1) e (T1xT5) $p<0,05$

NIPS (TOxT1xT5): K-Wallis one-way $\mathrm{p}<0,001$

NIPS (TOxT1) e (T1xT5) $p<0,05$

Tabela 4 - Número de RN com e sem alteração dos parâmetros fisiológicos e comportamentais de acordo com o momento estudado

\begin{tabular}{|c|c|c|c|c|c|}
\hline \multirow[t]{2}{*}{ Variáveis fisiológicas } & \multicolumn{3}{|c|}{ NIPS $<3$} & \multicolumn{2}{|c|}{ NIPS $>3$} \\
\hline & T0 & T1 & T5 & T0 T1 & T5 \\
\hline \multicolumn{6}{|l|}{ Freqüência cardíaca } \\
\hline Com alteração & 7 & 11 & 9 & 421 & 17 \\
\hline Sem alteração & 22 & 16 & 16 & 172 & 8 \\
\hline \multicolumn{6}{|l|}{ Freqüência respiratória } \\
\hline Com alteração & 12 & 7 & 3 & 18 & 3 \\
\hline Sem alteração & 35 & 22 & 29 & 213 & 15 \\
\hline \multicolumn{6}{|l|}{ Saturação de oxigênio } \\
\hline Com alteração & & 2 & 2 & 6 & 2 \\
\hline Sem alteração & 45 & 28 & 25 & $4 \quad 14$ & 10 \\
\hline
\end{tabular}

Os valores obtidos para a freqüência cardíaca, a freqüência respiratória e a saturação de oxigênio foram expressos em média, e os da escala de NIPS foram expressos em mediana e estão apresentados na Tabela 2.

Os resultados não mostraram diferenças estatisticamente significantes das freqüências cardíaca e respiratória, nos três momentos estudados (ANOVA one-way-FC: $\mathrm{p}=0,447 ; \mathrm{FR}: \mathrm{p}=0,864$ ).

Quanto à saturação arterial de oxigênio, verificou-se diferença significativa entre os três momentos estudados (K-Wallis one-way analysis of variance on ranks $\mathrm{p}<0,001$; comparações múltiplas Dunn `s Method-T0x T1 e T1 x T5: p < 0,05).

Com relação aos resultados obtidos com a aplicação da NIPS (Tabela 2), a mediana dos escores observados no T0, T1 e T5 apresentaram significância estatística, sendo o escore da mediana maior no momento imediatamente após a aspiração endotraqueal (K-Wallis one-way analysis of variance on ranks $\mathrm{p}<0,001$; comparações múltiplas Dunn `s Method - T0 x T1 e T1 x T5: p < 0,05).

Com relação à presença de bradicardia ou taquicardia no momento $\mathrm{T} 1,46 \%$ dos RN apresentaram bradi/taquicardia. Quanto à bradipnéia ou taquipnéia não houve diferença do número de pacientes nos três tempos do estudo. Em relação à presença de hipóxia, o momento imediatamente após aspiração endotraqueal, um maior número de pacientes apresentou saturação inferior a 90\% (Tabela 3).

Quanto à presença de dor, avaliada por meio da aplicação da escala de NIPS (Tabela 3), nos tempos 1 e 5, um número significantemente maior de pacientes apresentou NIPS superior a três, sendo superior o número de pacientes que apresentaram dor imediatamente após a aspiração endotraqueal.

Com relação à presença ou não de alteração das variações fisiológicas e comportamentais em cada momento estudado (Tabela 4), observou-se um maior número de pacientes que apresentou alteração na frequiência cardíaca com escore de NIPS superior a três, imediatamente após a aspiração endotraqueal, e um número expressivo de pacientes sem alteração na freqüência cardíaca e com NIPS menor que três. Quanto à frequiência respiratória, a maioria dos pacientes não demonstrou alteração com o escore de NIPS inferior a três no momento T1, enquanto que um número menor de pacientes apresentou alteração na freqüência respiratória com NIPS superior a três em T1. Na saturação de oxigênio 28 pacientes com NIPS inferior a três não apresentaram alteração imediatamente após aspiração endotraqueal, enquanto que apenas seis pacientes, tendo um escore de NIPS superior a três, apresentaram alteração.

Em relação à sensibilidade e à especificidade das variáveis fisiológicas (Tabela 5), a freqüência cardíaca foi a que apresentou maior sensibilidade, porém, foi a de menor especificidade. Já a saturação de oxigênio foi a que apresentou a menor sensibilidade para identificar a presença de dor, porém foi a mais específica. Em relação as variáveis comportamentais, apresentadas por NIPS $>3$, tanto a sensibilidade quanto a especificidade obtidas foram altas, sendo a especificidade de $86,6 \%$. 


\section{Discussão}

O avanço tecnológico na área neonatal tem permitido a sobrevida de prematuros cada vez menores. Assim, esta população é exposta rotineiramente à dor e aos seus efeitos deletérios durante seu longo período de internação. Embora haja um número crescente de publicações abordando a dor neonatal, poucos foram os progressos que efetivamente trouxeram mudanças nas Unidades de Terapia Intensiva Neonatais, no que se refere à implementação de intervenções específicas para reduzir os efeitos do estresse e os estímulos dolorosos ${ }^{1}$.

Os mecanismos nociceptivos iniciam seu desenvolvimento a partir do segundo e terceiro trimestres de gestação. O desenvolvimento prossegue após o nascimento até os dois anos de idade. A percepção nociceptiva varia pouco em relação à vida adulta, apesar das grandes mudanças nos aspectos emocionais da experiência dolorosa, das interpretações do significado da dor e do comportamento e expressão cognitiva diante da dor. Já houve tempo no qual se acreditou que o sistema de percepção à dor seria subdesenvolvido no recém-nascido e lactente. Esta idéia, todavia, foi resultado da racionalização conceitual com base na incapacidade que crianças, neste estágio do desenvolvimento, têm de verbalizar os fenômenos subjetivos da dor ${ }^{9}, 10$.

Atualmente não resta dúvida que a densidade das terminações nervosas cutâneas dos recém-nascidos e lactentes é idêntica à de pacientes adultos. Pesquisas microneurográficas apontam que as propriedades neurofisiológicas dos receptores nociceptivos são similares entre adultos e crianças. Da mesma forma, já ficou demonstrado que as respostas despolarizantes de mecanoceptores e receptores de pressão a estímulos mecânicos, químicos e a mediadores inflamatórios são iguais àquelas observadas em adultos. Recém-nascidos exibem reflexos complexos e muito bem desenvolvidos, o que pressupõe um grau razoável de organização do corno dorsal medular. A partir da metade do terceiro trimestre da gestação pode-se observar a liberação de dopamina e noradrenalina após estímulo sensorial, comprovando que as vias inibitórias descendentes dos centros supra-espinhais já estão desenvolvidas e maduras. O que se admite hoje, na verdade, é que recém-nascidos e lactentes têm sensibilidade aumentada ao estímulo doloroso, pois o que permanece imaturo são alguns mecanismos moduladores, funcionando abaixo de sua capacidade plena, permitindo que o estímulo doloroso alcance estruturas centrais com mais intensidade ${ }^{9-11}$.

Para se aferir a dor em um recém-nascido, dispõe-se de parâmetros fisiológicos e comportamentais. Dentre os fisiológicos destacam-se: frequiências cardíaca e respiratória, pressão arterial, saturação de oxigênio e dosagens hormonais, ligadas à resposta endócrino-metabólica, ${ }^{9}$. Dentre as alterações comportamentais, a mímica facial do recém-nascido é sensível e útil na prática clínica diária.

Com base nas alterações comportamentais, várias escalas foram criadas e validadas ${ }^{8,11-12}$. Estes instrumentos apresentam como vantagem a sua fácil aplicabilidade, no entanto, pela falta de objetividade eles dependem da interpretação do observador acerca dos comportamentos avaliados.
Tabela 5 - Sensibilidade, especificidade e valores preditivos positivos e negativos dos parâmetros fisiológicos e comportamentais na população estudada

\begin{tabular}{lcccc}
\hline & S & E & VPP & VPN \\
\hline Freqüência Cardíaca & $40,7 \%$ & $8,6 \%$ & $34,3 \%$ & $11,1 \%$ \\
Freqüência Respiratória & $24,1 \%$ & $61,9 \%$ & $46,6 \%$ & $37,1 \%$ \\
Saturação de oxigênio & $6,6 \%$ & $70 \%$ & $25 \%$ & $33,3 \%$ \\
Escala de NIPS & $65,7 \%$ & $86,6 \%$ & $92 \%$ & $52 \%$ \\
\hline
\end{tabular}

S: sensibilidade; E: especificidade; VPP: valores preditivos positivos; VPN: valores preditivos negativos

Recentes estudos têm utilizado tecnologia de ponta para aprimorar a avaliação da dor em recém-nascidos. Um exemplo disto é o 'uso da espectroscopia por intravermelho, que mensura a oxigenação tissular. Esta técnica é amplamente utilizada em âmbito experimental, porém, ainda é muito restrita na clínica do dia-a-dia e muito pouco estudada para recém-nascidos prematuros ${ }^{13}$.

O presente estudo visou verificar a especificidade e a sensibilidade das variáveis fisiológicas e comportamentais, como instrumentos de avaliação da dor aguda em recém-nascidos pré-termos, sob ventilação mecânica no momento da aspiração endotraqueal.

Os dados encontrados em nosso estudo são semelhantes aos da literatura, em relação aos parâmetros fisiológicos, observando-se aumento da FR e da FC. Conforme Guinsburg et al., dentre as respostas mais conhecidas do organismo humano ao estímulo doloroso agudo, destaca-se a taquicardia² ${ }^{2}$.

Neste estudo não foram observadas alterações significativas da FC e FR nos três momentos estudados, o que indica que o uso da freqüência cardíaca e respiratória isoladamente para a avaliação da dor, não se mostrou válida para identificar recém-nascidos pré-termos submetidos a um procedimento doloroso.

De acordo com Santos et al., a FC, como parâmetro isolado, é de difícil avaliação, pois é inespecífica e, além disso, o fato de seus valores situarem-se dentro dos limites aceitos como normais, não alerta o profissional sobre a possibilidade de o RN estar sentindo dor ${ }^{1}$.

Em relação à saturação de oxigênio, houve diferença estatística entre os três momentos estudados. Dessa maneira, a saturação de oxigênio parece válida para a avaliação da dor do recém-nascido prétermo, pois houve queda consistente e acentuada de seus valores logo após o estímulo doloroso. No entanto, segundo Lübe et al., sabe-se que ela é inespecífica, uma vez que se altera diante de qualquer estímulo desagradável ${ }^{5}$.

A hipoxemia apresentada pelos recém-nascidos no primeiro minuto após o estímulo doloroso ocorre não só pela presença de dor, mas também devido ao procedimento de escolha, aspiração endotraqueal, em que se exerce uma pressão negativa na via aérea do recém-nascido. Este procedimento pode acarretar efeitos indesejáveis, tais como trauma da mucosa traqueo-brônquica, hipoxia, bradicardia, atelectasias e aumento da pressão intra-craniana ${ }^{18,19}$. 
Quanto à aplicação da NIPS em nosso estudo, um número maior de recém-nascidos mostraram pontuação da escala de dor superior a 3 no primeiro e no quinto minuto após serem submetidos à aspiração endotraqueal; o que foi confirmado por Guinsburg et al., constatando que a escala de NIPS é um instrumento sensível e específico para a avaliação da dor do recém-nascido pré-termo. Entretanto, segundo os autores são necessários mais dados a respeito do comportamento da NIPS em prematuros criticamente doentes?

Foi observada divergência nos resultados entre a presença de dor com a utilização da escala de avaliação da dor e os parâmetros fisiológicos, já que nos momentos T1 e T5 a NIPS indicou presença de dor, enquanto que as variáveis fisiológicas não apresentaram alterações significantes. Dados também relatados na literatura, ressaltando que na presença da dor ocorrem movimentos expiratórios forçados, taquicardia, oscilações no suprimento de oxigênio ao cérebro e no fluxo sanguíneo cerebral, além de outras manifestações metabólicas, porém sem alterações significativas ${ }^{18,20}$.

As variáveis fisiológicas apresentam pouca sensibilidade e especificidade para a avaliação da dor no RN prematuro quando avaliadas isoladamente. A utilização simultânea de parâmetros fisiológicos e comportamentais sugere ser a forma mais adequada para a detecção da dor em recém-nascidos prematuros.

\section{Referências}

1. Santos JA, Procianoy RS, Bohrer BB, Noer C, Librelato GA, Campelo JN. Os recém-nascidos sentem dor quando submetidos à sondagem gástrica? J Pediatr (Rio J) 2001;77(5):374-80.

2. Guinsburg R, Kopelman BI, Almeida MF, Miyoshi MH. A dor do recémnascido prematuro submetido à ventilação mecânica através de cânula traqueal. J Pediatr (Rio J) 1994;70(2):82-90.

3. Guinsburg R. A linguagem da dor no recém-nascido. Jornal da Paulista 2000;139:145-9.

4. Silva YP, Silva JF, Costa LP, Medeiros MF, Mota JC. Avaliação da dor na criança. Rev Med Minas Gerais 2004;14:S92-S6.

5. Lübe A, Pereira T, Guinsburg R, Almeida M, Monteiro A, Santos A, Kopelman B. Validity of behavioral and physiologic parameters for acute pain assessment of term newborn infants. Sao Paulo Med J 1999;117(2):72-80.

6. Guinsburg R. Avaliação e tratamento da dor no recém-nascido. J Pediatr (Rio J) 1999;75:149-60.

7. Guinsburg R, Balda RC, Berenguel RC, Almeida MF, Tonelotto J, Santos AM et al. Aplicação das escalas comportamentais para a avaliação da dor em recém-nascidos. J Pediatr 1997;73(6):411-7.

8. Branco A, Fekete WMS, Rugolo SSML, Rehder IM. Valor e variações da frequência fundamental no choro de dor de recém-nascidos. Rev Cefac 2006;8(4):529-35.

9. Sweet SD, McGrath PJ. Physiological measures of pain. In: Finley GA, McGrath PJ. Measurement of pain in infants and children. IASP Press: Seattle 1997. p. 59-81.

10. Ranger M, Johnston CC, Anand KJ. Current controversies regarding pain assessment in neonates. Semin Perinatol 2007;31(5):283-8.
11. Cameron EC, Raingangar V, Khoori N. Effects of handling procedures on pain responses of very low birth weight infants. Pediatr Phys Ther 2007;19(1):40-7.

12. Chermont AG, Guinsburg R, Balda RC, Kopelman BI. 0 que os pediatras conhecem sobre avaliação e tratamento da dor no recém-nascido? J Pediatr (Rio J) 2003;79(3):265-72.

13. Slater R, Fitzgerald M, Meek J. Can cortical responses following noxius stimulation inform us about pain processing in neonates? Semin Perinato 2007;31(5):298-302.

14. Espinosa AG, Mendoza AR. Dolor en recién nacidos. Acta Pediatr Mex 1997;18(2):94-5.

15. Departamento de Pediatria da Universidade Federal do Paraná. Sedação e analgesia em recém-nascidos. In: Departamento de Pediatria da UFPR. Manual de Neonatologia $3^{\mathrm{a}}$ Ed. Curitiba: UFPR: 2001.

16. Reichert AP, Silva SL, Oliveira JM. Dor no recém-nascido: uma realidade a ser considerada. Nursing 2000;30:28-30.

17. Souza F. Dor: o quinto sinal vital. Rev Latino-Am Enf 2002;10(3):446-7.

18. Nicolau CM, Yasukawa AS. Abordagem da dor na assistência fisioterapêutica. In: Silva APA, Forte MJP, Juliani RCTP, Azevedo SDR. Instituto da Criança 30 anos. São Paulo: Editora Yendis: 2006.

19. Garcia JM, Nicolau CM. Assistência fisioterápica aos recém-nascidos do berçário anexo à Maternidade do Hospital das Clínicas da Universidade de São Paulo. Rev Fisioter Univ Säo Paulo 1996;3(1/2):38-46.

20. Nicolau CM. Estudo das repercussões da fisioterapia respiratória sobre a função cardiopulmonar em recém-nascidos pré-termo de muito baixo peso. [Dissertação de Mestrado]. São Paulo: Faculdade de Medicina da Universidade de São Paulo; 2006. 